\title{
ANALYTICITY AND QUASI-ANALYTICITY FOR ONE-PARAMETER SEMIGROUPS
}

\author{
J. W. NEUBERGER ${ }^{1}$
}

AbSTRACt. Suppose that $T$ is a strongly continuous (even at 0 ) one-parameter semigroup of bounded linear transformations on a real Banach space $S$ and $T$ has generator $A$.

Theorem A. If lim $\sup _{x \rightarrow 0}|T(x)-I|<2$ then $A T(x)$ is bounded for all $x>0$.

Suppose $\left\{\delta_{q}\right\}_{q=1}^{\infty}$ is a sequence of positive numbers convergent to 0 and each of $N(q), q=1,2, \cdots$ is an increasing sequence of positive integers. Denote by $Q$ the collection consisting of (1) all real analytic functions on $(0, \infty)$ and (2) all $h$ on $(0, \infty)$ for which there is a Banach space $S$, a member $p$ of $S$, a member $f$ of $S^{*}$ and a strongly continuous semigroup $L$ of bounded linear transformations so that $h(x)=f[L(x) p]$ for all $x>0$ where $L$ satisfies $\lim \sup _{n \rightarrow \infty}(n \in N(q))\left|L\left(\delta_{q} / n\right)-I\right|<2, q=1,2, \cdots$. $(0, \infty)$.

THEOREM B. No two members of $Q$ agree on an open subset of

1. Introduction and statement of theorems. Suppose that $S$ is a real Banach space and $T$ is a strongly continuous (even at 0 ) oneparameter semigroup of bounded linear transformations from $S$ to $S$. If $p$ is in $S$, then the function $g_{p}$ so that $g_{p}(x)=T(x) p$ for all $x \geqq 0$ is called a trajectory of $T$ and, if $f$ is in $S^{*}$, a function $h$ so that $h(x)=f\left(g_{p}(x)\right)$ for all $x>0$ will be referred to as a functional of a trajectory of $T$.

For comparison with the following theorem recall that if $\lim _{x \rightarrow 0}|T(x)-I|=0$, then the generator $A$ of $T$ is bounded and each functional of a trajectory of $T$ is analytic $[4, \S 9.4]$.

Theorem A. If

$$
\limsup _{x \rightarrow 0}|T(x)-I|<2,
$$

then $A T(x)$ is bounded for all $x>0$.

Presented to the Society, November 21, 1969 under the title Analyticity and quasianalyticity of trajectories of semigroups of bounded linear transformations; received by the editors November 24, 1969.

AMS Subject Classifications. Primary 4750.

Key Words and Phrases. Quasi-analytic, analytic, semigroup of bounded linear transformations.

${ }^{1}$ Written while the author was an Alfred P. Sloan Research Fellow. Also supported in part by a research grant from Emory University. 
Corollary. If $T$ satisfies (1) and $T$ can be extended to a group of bounded linear transformations, then $A$ is bounded.

As a consequence of the next theorem, one has that if $T$ satisfies instead of (1) the weaker condition

$$
\limsup _{n \rightarrow \infty}\left|T\left(3^{-n}\right)-I\right|<2
$$

and each of $h$ and $k$ is a functional of a trajectory of $T$, then $h$ and $k$ do not agree on an open subset of $(0, \infty)$ unless $h=k$. Moreover, if $r$ is an analytic function on $(0, \infty)$ and $h$ is a functional of a trajectory of $T$, then $r$ and $h$ do not agree on an open subset of $(0, \infty)$ unless $r=h$.

Using the first example in $\$ 3$ one can find a semigroup satisfying (2) so that some functional of one of its trajectories is not analytic.

Before the next theorem is stated some preliminary definitions are given. Denote by $\Delta=\left\{\delta_{q}\right\}_{q=1}^{\infty}$ a sequence of positive numbers converging to 0 and denote by $K=\{N(q)\}_{q=1}^{\infty}$ a sequence each term of which is an increasing sequence of positive integers $\left(N(q)=\left\{n_{q, j}\right\}_{j=1}^{\infty}, q=1,2, \cdots\right)$. Call a strongly continuous semigroup $M$ on a real Banach space $(\Delta, K)$-regular if

$$
\limsup _{j \rightarrow \infty}\left|M\left(\delta_{q} / n_{q, j}\right)-I\right|<2, \quad q=1,2, \cdots .
$$

Denote by $Q(\Delta, K)$ the collection to which $h$ belongs if and only if $h$ is a functional of a trajectory of a $(\Delta, K)$-regular semigroup.

THEOREM B. $Q(\Delta, K)$ is a quasi-analytic collection in the sense that no two members of it agree on an open subset of $(0, \infty)$. Moreover, if $h$ is an analytic function on $(0, \infty)$ and a member $k$ of $Q(\Delta, K)$ agrees with $h$ on an open subset of $(0, \infty)$, then $h=k$.

It is known $[4, \S 19.4]$ that there is a strongly continuous semigroup so that some functional of one of its trajectories is not identically zero on $(0, \infty)$ but is zero on some open subset of $(0, \infty)$. Such a function is not in any of the collections $Q(\Delta, K)$. An example is given in $\S 3$ which shows that for $\Delta$ and $K$ properly chosen, $Q(\Delta, K)$ contains a nonanalytic member.

The problem of extending the present development to nonlinear semigroups is illustrated by an example.

Kato [2] has a theorem from which Theorem A follows as a very special case. Williams [9] arrived at the corollary to Theorem A before Theorem A was found. In about 1965 Kendall [3], using [5], 
showed that functionals of trajectories of semigroups satisfying (1) formed a quasi-analytic collection. Independently the present writer obtained the weaker result (announced in [6] but never published) that on a given space, the collection of all trajectories of semigroups satisfying (1) form a quasi-analytic collection.

The proof of Theorem A given in this note depends heavily on the following result of Beurling [1] to which this author has had access since early 1968.

Suppose $f$ is a continuous real-valued function on $[-4,4]$ and that for some $\rho$ in $[3 / 2,2)$,

$$
\left|\sum_{q=0}^{n}\left(\begin{array}{l}
n \\
q
\end{array}\right)(-1)^{n-q} f(u+q(v-u) / n)\right| \leqq \rho^{n}
$$

if $u$ and $v$ are in $[-4,4], n=1,2, \ldots$. Then $f$ can be extended analytically to the rhombus with vertices at $\pm 4, \pm 4 i k \alpha^{2}$ where $\alpha=(2-\rho) / 4$.

One can get from Beurling's argument that the following is true: Suppose that $t$ is a number, $\delta^{\prime}>0$ and $G$ is a collection of real-valued continuous functions whose domains include $\left[t-\delta^{\prime}, t+\delta^{\prime}\right]$ so that for some $L>0$ and $\rho$ in $[3 / 2,2)$,

$$
\left|\sum_{q=0}^{n}\left(\begin{array}{l}
n \\
q
\end{array}\right)(-1)^{n-q} f(u+q(v-u) / n)\right| \leqq L \rho^{n},
$$

for all $u, v$ in $\left[t-\delta^{\prime}, t+\delta^{\prime}\right]$ and all $f$ in $G, n=0,1, \cdots$, where $\sum_{q=0}^{0}$ above is $f(u)$. Then there exist $\delta, M>0$ so that if $f$ is in $G$, then the restriction of $f$ to $[t-\delta, t+\delta]$ has an analy tic extension $\hat{f}$ to the closure of the region $R_{\delta}(t) \quad(=\{z:|t-z|<\delta\})$ of the complex plane so that $|\hat{f}(z)| \leqq M$ for all $z$ in $\operatorname{cl}\left(R_{\delta}(t)\right)$.

Theorem $\mathrm{B}$ of this note follows from Theorem 2 of [8] which in turn depends on the following slight generalization (Lemma A of [8]) of Lemma 8 of [5]. Suppose that $u$ and $v$ are numbers, $c=(2 u / 3)+(v / 3)$ and $f$ is a continuous real-valued function whose domain includes $[u, v]$ such that (1) $f(x)=0$ if $x$ is in $[u, c]$ and (2) if $y$ is in $(c, v]$ then there is a number $x$ in $[c, y]$ such that $f(x) \neq 0$. Then

$$
\lim _{n \rightarrow \infty}\left[\sum_{s=0}^{n}\left(\begin{array}{l}
n \\
s
\end{array}\right)\left|\sum_{t=0}^{s}\left(\begin{array}{l}
s \\
t
\end{array}\right)(-1)^{s-t} f(u+t(v-u) / n)\right|\right]^{1 / n}=3 .
$$

For earlier results concerning the approximation of the identity element by semigroups see $[4, \S 10.7]$. 


\section{Proofs.}

Proof of Theorem A. If $f$ is in $S^{*}$ and $p$ is in $S$, denote by $h_{p, f}$ the function on $(0, \infty)$ so that $h_{p, f}(x)=f[T(x) p]$ for all $x>0$. Denote by each of $\epsilon$ and $\delta^{\prime}$ a positive number so that $|T(x)-I| \leqq 2-\epsilon$ if $0 \leqq x \leqq 2 \delta^{\prime}$. Suppose $t>0$ and denote by $M^{\prime}$ a positive number so that $|\bar{T}(x)| \leqq M^{\prime}$ if $x$ is in $J=\left[t-\delta^{\prime}, t+\delta^{\prime}\right]$. Denote by $G$ the collection of all functions $h_{p, f}$ so that $\|p\| \leqq 1,|f| \leqq 1$. If each of $u$ and $v$ is in $J$, $u<v, n$ a nonnegative integer and $h_{p, f}$ is in $G$, then

$$
\begin{aligned}
&\left|\sum_{q=0}^{n}\left(\begin{array}{l}
n \\
q
\end{array}\right)(-1)^{n-q} h_{p, f}(u+q(v-u) / n)\right| \\
&\left.=\mid f\left[\sum_{q=0}^{n}\left(\begin{array}{l}
n \\
q
\end{array}\right)(-1)^{n-q}(T(v-u) / n)\right)^{q} T(u) p\right] \mid \\
& \leqq|f||T(u)|\|p\||T((v-u) / n)-I|^{n} \leqq M^{\prime}(2-\epsilon)^{n} .
\end{aligned}
$$

By the comment following the statement of Beurling's theorem, there is a positive number $M$ and a positive number $\delta$ so that if $h_{p, f}$ is in $G$, then the restriction of $h_{p, f}$ to $[t-\delta, t+\delta]$ has an analytic extension $\hat{h}_{p, f}$ to $\operatorname{cl}\left(R_{\delta}(t)\right)$ such that $\left|\hat{h}_{p, f}(z)\right| \leqq M$ for all $z$ in $\operatorname{cl}\left(R_{\delta}(t)\right)$. Hence there is a number $K$ so that $\left|h_{p, f}^{\prime}(x)\right| \leqq K$ for all $h_{p, f}$ in $G$ and $x$ in $[t-\delta / 2, t+\delta / 2]=J^{\prime}$. So, if $x$ is in $J^{\prime}, x \neq t$, and $h_{p, f}$ is in $G$, then

$$
\begin{aligned}
\left|f\left[(x-t)^{-1}(T(x)-T(t)) p\right]\right| & =\left|(x-t)^{-1}\left[h_{p, f}(x)-h_{p, f}(t)\right]\right| \\
& =\left|h_{p, f}^{\prime}\left(x_{0}\right)\right| \leqq K
\end{aligned}
$$

for some $x_{0}$ in $[x, t]$. Hence $\left\|(x-t)^{-1}(T(x)-T(t)) p\right\| \leqq K$ for all $x$ in $J^{\prime}$ different from $t$ and all $p$ in $S$ such that $\|p\| \leqq 1$. But this implies that $\left\{\left|(x-t)^{-1}(T(x)-T(t))\right|: 0<|x-t|<\delta / 2\right\}$ is bounded. Since $A$ is densely defined $[4, \S 10.3]$, then if $p$ is in the domain of $A$,

$$
\lim _{x \rightarrow t^{+}}(x-t)^{-1}(T(x)-T(t)) p=T(t) \lim _{x \rightarrow t^{+}}(x-t)^{-1}(T(x-t)-I) p=T(t) A p
$$

and so

$$
\lim _{x \rightarrow t^{+}}(x-t)^{-1}(T(x-t)-I) T(t) p=\lim _{x \rightarrow t^{+}}(x-t)^{-1}(T(x)-T(t)) p
$$

exists and is equal to $A T(t) p$. Hence if $x_{1}, x_{2}, \cdots$ is a decreasing sequence of members of $J^{\prime}$ which converges to $t$ then $\left\{\left(x_{n}-t\right)^{-1}\left(T\left(x_{n}\right)-T(t)\right)\right\}_{n=1}^{\infty}$ converges pointwise on a dense subset of $S$ to $A T(t)$. From the Banach-Steinhaus theorem it follows that $\left\{\left(x_{n}-t\right)^{-1}\left(T\left(x_{n}\right)-T(t)\right)\right\}_{n=1}^{\infty}$ converges pointwise on all of $S$ to a bounded linear transformation. Such a transformation is a bounded 
extension of $A T(t)$. It follows that $A T(t)$ is bounded and since $A T(t)$ is closed it must have domain all of $S$.

Proof of Theorem B. Suppose $h$ is a member of $Q(\Delta, K)$. Then for some real Banach space $S$ there is a point $p$ in $S$, a member $f$ of $S^{*}$ and a strongly continuous semigroup $T$ on $S$ so that $h(x)=f[T(x) p]$ for all $x>0$. As in the proof of the preceding theorem if $u, v>0, n$ is a positive integer and $r$ is a positive integer $\leqq n$, then if $a=|v-u|$ and $M=1$. u.b. ${ }_{x \in[u, v]}|T(x)|$

$$
\left|\sum_{s=0}^{r}\left(\begin{array}{l}
r \\
s
\end{array}\right)(-1)^{r-s} h(u+s(v-u) / n)\right| \leqq|f| M\|p\||T(a / n)-I|^{r}
$$

and so

$$
\begin{aligned}
D_{h}(n ; u, v) & \equiv \sum_{r=0}^{n}\left(\begin{array}{l}
n \\
r
\end{array}\right)\left|\sum_{s=0}^{r}\left(\begin{array}{l}
r \\
s
\end{array}\right)(-1)^{r-s} h(u+s(v-u) / n)\right| \\
& \leqq|f| M\|p\|[1+|T(a / n)-I|]^{n} .
\end{aligned}
$$

If for some positive integer $q,|v-u|=\delta_{q}$, then

$$
\begin{aligned}
\limsup _{n \rightarrow \infty} \sup _{(n \in N(\ell))} & {\left[D_{h}(n: u, v)\right]^{1 / n} } \\
& \leqq \\
& \lim _{n \rightarrow \infty} \sup _{(n \in N(q))}|f|^{1 / n} M^{1 / n}\|p\|^{1 / n}\left[1+\left|T\left(\delta_{q} / n\right)-I\right|\right]<3
\end{aligned}
$$

since $\lim \sup _{j \rightarrow \infty}\left|T\left(\delta_{q} / n_{q, j}\right)-I\right|<2$ and $N(q)=\left\{n_{q, j}\right\}_{j=1}^{\infty}$. Hence according to Theorem 2 of [8], $Q(\Delta, K)$ is a quasi-analytic collection.

Suppose now that $k$ is an analytic function on $(0, \infty)$ and $k$ agrees on some open subset of $(0, \infty)$ with a function $h$ in $Q(\Delta, K)$. Denote $h-k$ by $g$, denote by $q$ a positive integer and denote by $u, v$ and $w$ three positive numbers so that $v=(2 u / 3)+(w / 3),|u-w|=\delta_{q}$ and so that $g(x)=0$ if $x$ is in $[u, v]$ but, if $y$ is in $(v, w]$, then there is a number $y^{\prime}$ in $[v, y]$ so that $g\left(y^{\prime}\right) \neq 0$. By Lemma A of [8], $\lim _{n \rightarrow \infty} D_{o}(n ; u, w)^{1 / n}=3$. This leads to a contradiction since if $n$ is a positive integer, $D_{o}(n: u, w)=D_{h-k}(n ; u, w) \leqq D_{h}(n ; u, w)$ $+D_{k}(n ; u, w)$ and so

$$
\begin{aligned}
& \quad \limsup _{n \rightarrow \infty} \sup _{(n \in N(q))}(n ; u, w)^{1 / n} \\
& \leqq \max \left\{\limsup _{n \rightarrow \infty} D_{(n \in N(q))} D_{h}(n ; u, w)^{1 / n}, \lim _{n \rightarrow \infty} \sup _{(n \in N(q))} D_{k}(n ; u, w)^{1 / n}\right\}<3 .
\end{aligned}
$$

So Theorem B is established. To get an analagous theorem replace the word "real" in each instance by "complex" in the introduction to and proof of Theorem B. 
3. Examples. In real $l_{2}$ define $T$ so that if $\left(a_{1}, b_{1}, a_{2}, b_{2}, \cdots\right)$ is in $l_{2}$, then

$$
\begin{aligned}
& T(x)\left(a_{1}, b_{1}, a_{2}, b_{2}, \cdots\right) \\
& =\left(a_{1} \cos x-b_{1} \sin x, a_{1} \sin x+b_{1} \cos x,\right. \\
& \left.\quad a_{2} \cos 2 x-b_{2} \sin 2 x, a_{2} \sin 2 x+b_{2} \cos 2 x, \cdots\right)
\end{aligned}
$$

for all $x \geqq 0$. Suppose that $0<\epsilon<1 / 2, \Delta=\left\{\delta_{q}\right\}_{q=1}^{\infty}$ and $K=\{N(q)\}_{q=1}^{\infty}$ $=\left\{\left\{n_{q, j}\right\}_{j=1}^{\infty}\right\}_{q=1}^{\infty}$ are chosen so that

$$
\min _{t=0,1,2, \ldots}\left|\left(p \delta_{q} / 2 \pi n_{q, j}\right)-t\right| \leqq \epsilon, \quad q, j=1,2, \cdots,
$$

holds for infinitely many positive integers $p$. Denote by $\left\{k_{p}\right\}_{p=1}^{\infty}$ the sequence so that if $p$ is a positive integer $k_{p}=1$ if (4) holds and $k_{p}=0$ if (4) does not hold. Denote by $l_{2}^{\prime}$ the subspace consisting of all points $\left(a_{1} k_{1}, b_{1} k_{1}, a_{2} k_{2}, b_{2} k_{2}, \cdots\right)$ so that $\left(a_{1}, b_{1}, a_{2}, b_{2}, \cdots\right)$ is in $l_{2}$. Denote by $M$ the restriction of $T$ to $l_{2}^{\prime}$. Clearly $M$ is strongly continuous on $l_{2}^{\prime}$. Some calculation shows that

$$
\limsup _{j \rightarrow \infty}\left|M\left(\delta_{q} / n_{q, j}\right)-I\right|<(2(1-\cos 2 \pi \epsilon))^{1 / 2}, \quad q=1,2, \cdots,
$$

so that all functionals of trajectories of $M$ are in $Q(\Delta, K)$. But, if $P=\left(a_{1} k_{1}, \quad b_{1} k_{1}, \quad a_{2} k_{2}, \quad b_{2} k_{2}, \cdots\right)$ where $a_{p}=(p+1)^{-3 / 4}, \quad b_{p}=0$, $p=1,2, \cdots$, and $h(x)=(M(x) P, P)$ for all $x>0$, then $h(x)$ $=\sum_{p=1}^{\infty} k_{p}(p+1)^{-3 / 2} \cos p x$ for all $x>0$. Since for infinitely many positive integers $p, k_{p}=1$, it must be that $h$ does not have a continuous second derivative on $(0, \infty)$ and so (see proof of Theorem 2 of $[8])$ is certainly not analytic on $(0, \infty)$. This shows that the hypothesis of Theorem B does not imply the hypothesis of Theorem A.

For the second example, consider the strongly continuous nonlinear semigroup on $E_{1}$ given in [7]:

$$
\begin{aligned}
T(x)=p-x & \text { if } p \geqq 1 \text { and } p-x \geqq 1, x \geqq 0, \\
=1 & \text { if } p \geqq 1 \text { and } p-x<1, x>0, \\
=p & \text { if } p<1, x \geqq 0 .
\end{aligned}
$$

If $p$ is a number define $g_{p}(x)=T(x) p$ for all $x>0$. Then if $p>1$, $g_{p} \neq g_{1}$ but $g_{p}(x)=g_{1}(x)$ for all $x \geqq p-1$. However, if $x>0$, the Lipshitz norm of $T(x)-I,|T(x)-I|$, is equal to 1 . One expects, nevertheless, interesting generalizations of Theorems A and B to semigroups of nonlinear transformations. 


\section{REFERENCES}

1. A. Beurling, On analytic extension of semi-groups of operators, J. Functional Analysis (to appear).

2. T. Kato, A characterization of holomorphic semigroups, Proc. Amer. Math. Soc. (to appear).

3. D. G. Kendall, Some recent developments in the theory of denumerable Markov processes, Trans. Fourth Prague Conf. on Information Theory, Statistical Decision Functions, Random Processes (Prague, 1965), Academia, Prague, 1967, pp. 11-27. MR 36 \#974.

4. E. Hille and R. S. Phillips, Functional analysis and semi-groups, rev. ed., Amer. Math. Soc. Colloq. Publ., vol. 31, Amer. Math. Soc., Providence, R.I., 1957. MR 19, 664.

5. J. W. Neuberger, A quasi-analyticity condition in terms of finite differences, Proc. London Math. Soc. (3). 14 (1964), 245-259. MR 28 \#3130.

6. - Quasi-analyticity of trajectories of semi-groups of bounded linear transformations, Notices Amer. Math. Soc. 12 (1965), 815. Abstract \#65T-454.

7. — An exponential formula for one-parameter semi-groups of nonlinear transformations, J. Math. Soc. Japan 18 (1966), 154-157. MR 34 \#622.

8. - Quasi-analytic collections containing Fourier series which are not infinitely differentiable, J. London Math. Soc. 43 (1968), 612-616. MR 37 \#5344.

9. D. Williams, On operator semigroups and Markov groups, (to appear).

Emory University, Atlanta, Georgia 30322 